\title{
FIRST SURVEY OF THE DRAGONFLY FAUNA (INSECTA, ODONATA) OF NORTHWESTERN BOSNIA
}

\section{Dejan Kulijer}

National Museum of Bosnia and Herzegovina, Zmaja od Bosne 3, 71000 Sarajevo, Bosnia and Herzegovina (e-mail: dejan.kulijer@gmail.com)

Kulijer, D.: First survey of the dragonfly fauna (Insecta, Odonata) of northwestern Bosnia. Vol. 26, No. 1., 65-80, 2017, Zagreb.

The results of the first study of the dragonfly fauna in northwest Bosnia are presented. Field surveys were conducted in summer 2012 and 2013, resulting in the identification of 36 species. Out of these, 34 are new for the region. The present paper gives a detailed overview of the results and a comparison of the dragonfly fauna composition of the region studied with the neighboring areas within Bosnia and Herzegovina and Croatia is made. The most abundant species were: Calopteryx virgo (Linnaeus, 1758), Sympetrum sanguineum (Müller, 1764), Onychogomphus forcipatus (Linnaeus,1758), Somatochlora meridionalis Nielsen, 1935, Calopteryx splendens (Harris, 1782) and Platycnemis pennipes (Pallas, 1771). Noteworthy are the records of Chalcolestes viridis (Vander Linden, 1825) and Coenagrion scitulum (Rambur, 1842), two species that are rare in Bosnia and Herzegovina, and also Coenagrion ornatum (Selys, 1850) and Cordulegaster heros Theischinger, 1979, species of European conservation concern. Distribution and the status of these species and the conservation of dragonfly species in the country are briefly discussed. The data collected represent an important contribution to the knowledge of the dragonfly fauna of the Una River basin and Bosnia and Herzegovina.

Key words: Bosnia and Herzegovina, distribution, insects, Coenagrion ornatum, Cordulegaster heros, Odonata, Una River.

Kulijer, D.: Prvi pregled faune vretenaca (Insecta, Odonata) sjeverozapadne Bosne. Vol. 26, No. 1., 65-80, 2017, Zagreb.

U ovom radu predstavljeni su rezultati istraživanja faune vretenaca sjeverozapadne Bosne. Terenskim istraživanjem koje je provedeno tijekom ljeta 2012. i 2013. godine zabilježeno je ukupno 36 vrsta vretenaca, među kojima su 34 vrste nove za ovo područje. U radu je prikazana detaljna analiza rezultata te usporedba sa sastavom faune vretenaca susjednih područja BiH i Hrvatske. Najzastupljenije zabilježene vrste su bile Calopteryx virgo (Linnaeus, 1758), Sympetrum sanguineum (Müller, 1764), Onychogomphus forcipatus (Linnaeus,1758), Somatochlora meridionalis Nielsen, 1935, Calopteryx splendens (Harris, 1782) i Platycnemis pennipes (Pallas, 1771). Među najznačajnije nalaze spadaju pronalasci vrsta Chalcolestes viridis (Vander Linden, 1825) i Coenagrion scitulum (Rambur, 1842), rijetkih vrsta u BiH, kao i vrsta od europskog konzervacijskog značaja, Coenagrion ornatum (Selys, 1850) i Cordulegaster heros Theischinger, 1979. Raspravljena su staništa i rasprostranjenost ovih vrsta u regiji i u BiH, te zaštita vretenaca u BiH.

Ključne riječi: Bosna i Hercegovina, Coenagrion ornatum, Cordulegaster heros, rasprostranjenost, kukci, Odonata, rijeka Una.

\section{INTRODUCTION}

Until recently, the Odonata fauna of Bosnia and Herzegovina $(\mathrm{B} \& \mathrm{H})$ received little attention and is poorly known (e.g. Kuljer et al., 2013, 2016). Only in the last decade were more comprehensive investigations undertaken, resulting in several papers with many new data and new species records for the country (e.g. BEDJANič, 2011; Jović et al., 
2010; Kulijer, 2012, 2014, 2015; Kulijer et al., 2012, 2013, 2016; Kulijer \& Topić, 2013; Kulijer \& Miljević, 2015). So far, 64 Odonata species have been recorded in Bosnia and Herzegovina (Kulijer et al., 2013; Kulijer \& Miljević, 2015), and the most comprehensive knowledge of Odonata fauna in Bosnia and Herzegovina has been provided by KuLIJER et al. (2013).

Although our knowledge of the dragonfly fauna of the country significantly improved in recent years, the occurrence of many species in several regions still remained largely unknown. The northwestern part of Bosnia is one of the least studied parts of the country for dragonflies. Odonatological surveys in this area have never been conducted and only some records of only two species from this region are known (KEROVEC, 2005a, 2005b). The largest part of this region belongs to the Una River basin, the catchment shared between Bosnia and Herzegovina and Croatia. The dragonfly fauna of this area is insufficiently known not only in Bosnia and Herzegovina, but also in Croatia (Franković \& Vilenica, 2009).

The aim of this study was to investigate dragonfly fauna of this region of Bosnia and Herzegovina for the first time and to determine species composition, their distribution and habitats, with a special focus on threatened species.

\section{MATERIALS AND METHODS}

\section{Study area}

The study area is the northwestern part of Bosnia and Herzegovina, located between $15^{\circ} 43^{\prime}$ and $16^{\circ} 32^{\prime}$ east, and $44^{\circ} 09^{\prime}$ and $45^{\circ} 13^{\prime}$ north. It encompasses the largest part of the Una River catchment, together with small parts of the Korana and Glina catchments that are situated within the borders of Bosnia and Herzegovina. The Una River is the main watercourse of the region. The Bosnian part of the upper Una River basin is situated in the mountainous region where the river flows through gorges and is often torrential (BoGNAR, 2005; International Sava River Basin Commission, 2009). In its lower reaches, particularly from the mouth of the Sana River, it changes into a mild and slow flowing river until its confluence with the Sava River (MatoničKin \& PAvletić, 1963). Apart from the Una River, major landscape features of the study area are Grmeč, Klekovača, Osječenica and Plješevica mountains. The southeastern part of the study region encompasses several karst poljes, including: Lušci polje, Petrovačko polje and Grahovsko polje. The lowest elevation in the study area is at the confluence of the Sana and Una rivers (117 $\mathrm{m}$ a.s.l.), while the highest point is at the top of the Klekovača mountain (1.961 m a.s.l.). The Una River valley, like other right tributaries' catchments within Croatia and Bosnia and Herzegovina, has a moderate continental climate. In the rivers' downstream sections there is also some influence of the Pannonian climate (International Sava River Basin Commission, 2009).

The Korana and Glina catchments (subcatchments of the Kupa River) are mainly situated in Croatia, with only a small part $\left(742 \mathrm{~km}^{2}\right)$ covering the northwestern corner of $\mathrm{B} \& \mathrm{H}$. This hilly region is also characterized by the presence of numerous small streams and rivers and the absence of larger stagnant water bodies (AnOnYmous, 2012). While the Una, Korana and Glina rivers all belong to the Black Sea Basin, the southernmost localities in the Bosansko Grahovo area (L. 72 - 75) belong to the Cetina River catchment of the Adriatic Sea Basin (Anonymous, 2012).

By its administrative organization, most of the area belongs to the Federation of Bosnia and Herzegovina (Unsko-Sanski Canton). The southernmost localities (L. 65 - 75) 
Tab. 1. List of localities with date of the survey, geographical coordinates In WGS84 and altitudes (m a.s.1.)

\begin{tabular}{|c|c|c|c|c|c|}
\hline No. & Locality name & Date & N latitude & E longitude & $\begin{array}{l}\text { Alti- } \\
\text { tude }\end{array}$ \\
\hline 1. & Kladušnica River, Velika Kladuša & 2005.11.02. & $45^{\circ} 10^{\prime} 59^{\prime \prime}$ & $15^{\circ} 49^{\prime} 13^{\prime \prime}$ & 140 \\
\hline 2. & Glinica River, Radiča Most, Vrnograč & 2013.07 .27 & $45^{\circ} 10^{\prime} 04^{\prime \prime}$ & $15^{\circ} 58^{\prime} 47^{\prime \prime}$ & 153 \\
\hline 3. & Stabandža Stream, Zborište 1 & 2013.07.27. & $45^{\circ} 08^{\prime} 28^{\prime \prime}$ & $16^{\circ} 01^{\prime} 45^{\prime \prime}$ & 185 \\
\hline 4. & Stabandža Stream, Zborište 2 & 2013.07.27. & $45^{\circ} 08^{\prime} 23^{\prime \prime}$ & $16^{\circ} 01^{\prime} 11^{\prime \prime}$ & 183 \\
\hline 5. & Kladušnica River, Mala Kladuša & 2012.08.19. & $45^{\circ} 06^{\prime} 57^{\prime \prime}$ & $15^{\circ} 51^{\prime} 57^{\prime \prime}$ & 168 \\
\hline 6. & Čaglica Stream, Pašin Brod & 2013.07.27. & $45^{\circ} 06^{\prime} 43^{\prime \prime}$ & $15^{\circ} 59^{\prime} 25^{\prime \prime}$ & 186 \\
\hline 7. & $\begin{array}{l}\text { Barjaktarevići, Polje, lake near old } \\
\text { manganise mine }\end{array}$ & 2013.07.27. & $45^{\circ} 06^{\prime} 26^{\prime \prime}$ & $16^{\circ} 02^{\prime} 00^{\prime \prime}$ & 236 \\
\hline 8. & Bužimica Stream, Varoška Rijeka & 2013.07.27. & $45^{\circ} 06^{\prime} 24^{\prime \prime}$ & $15^{\circ} 59^{\prime} 58^{\prime \prime}$ & 184 \\
\hline 9. & Pećina Stream, Donja Lučka & 2012.08 .19$. & $45^{\circ} 04^{\prime} 06^{\prime \prime}$ & $15^{\circ} 53^{\prime} 24^{\prime \prime}$ & 194 \\
\hline 10. & Čava Stream, Dobro selo & 2013.07.27. & $45^{\circ} 02^{\prime} 12^{\prime \prime}$ & $16^{\circ} 05^{\prime} 12^{\prime \prime}$ & 228 \\
\hline 11. & Una River, upstream from Bosanski Novi & 2011.07.12. & $45^{\circ} 01^{\prime} 58^{\prime \prime}$ & $16^{\circ} 21^{\prime} 32^{\prime \prime}$ & 120 \\
\hline 12. & Baštra Stream & 2013.07.27. & $45^{\circ} 01^{\prime} 39^{\prime \prime}$ & $16^{\circ} 04^{\prime} 13^{\prime \prime}$ & 239 \\
\hline 13. & Babinac Stream & 2013.07.27. & $45^{\circ} 00^{\prime} 57^{\prime \prime}$ & $16^{\circ} 07^{\prime} 06^{\prime \prime}$ & 197 \\
\hline 14. & Mutnica River, Tržac & 2005.11.02. & $44^{\circ} 59^{\prime} 42^{\prime \prime}$ & $15^{\circ} 47^{\prime} 16^{\prime \prime}$ & 257 \\
\hline 15. & Vodomut Stream & 2013.07.27. & $44^{\circ} 59^{\prime} 24^{\prime \prime}$ & $16^{\circ} 09^{\prime} 20^{\prime \prime}$ & 169 \\
\hline 16. & Donja Koprivna, stream and small lake & 2013.07.27. & $44^{\circ} 59^{\prime} 02^{\prime \prime}$ & $15^{\circ} 57^{\prime} 52^{\prime \prime}$ & 399 \\
\hline 17. & Small stream at Kapići, Cazin & 2012.08.19. & $44^{\circ} 58^{\prime} 55^{\prime \prime}$ & $15^{\circ} 52^{\prime} 13^{\prime \prime}$ & 270 \\
\hline 18. & Una River, Bosanska Otoka & $\begin{array}{l}\text { (a) 2005.07.26., } \\
\text { (b) 2005.11.02. }\end{array}$ & $44^{\circ} 57^{\prime} 35^{\prime \prime}$ & $16^{\circ} 10^{\prime} 55^{\prime \prime}$ & 140 \\
\hline 19. & Ravska Stream, Donji Agići & 2011.08 .06$. & $44^{\circ} 57^{\prime} 15^{\prime \prime}$ & $16^{\circ} 28^{\prime} 11^{\prime \prime}$ & 145 \\
\hline 20. & Gnjilovac Stream & 2013.07.27. & $44^{\circ} 57^{\prime} 12^{\prime \prime}$ & $15^{\circ} 57^{\prime} 23^{\prime \prime}$ & 376 \\
\hline 21. & Valovita Stream, Donji Agići & 2011.08.06. & $44^{\circ} 56^{\prime} 56^{\prime \prime}$ & $16^{\circ} 28^{\prime} 33^{\prime \prime}$ & 156 \\
\hline 22. & Ovanjska River, Marini & 2013.07.28. & $44^{\circ} 54^{\prime} 17^{\prime \prime}$ & $16^{\circ} 28^{\prime} 21^{\prime \prime}$ & 198 \\
\hline 23. & Una River, Ostrožac na Uni & 2012.08.19. & $44^{\circ} 54^{\prime} 15^{\prime \prime}$ & $15^{\circ} 56^{\prime} 57^{\prime \prime}$ & 191 \\
\hline 24. & Johovica Stream & 2013.07.28. & $44^{\circ} 53^{\prime} 59^{\prime \prime}$ & $16^{\circ} 21^{\prime} 37^{\prime \prime}$ & 208 \\
\hline 25. & Canyon of the Una River & 2013.07.27. & $44^{\circ} 53^{\prime} 57^{\prime \prime}$ & $16^{\circ} 02^{\prime} 20^{\prime \prime}$ & 187 \\
\hline 26. & Ostrožac, Cazin & 2012.05 .24 . & $44^{\circ} 53^{\prime} 52^{\prime \prime}$ & $15^{\circ} 56^{\prime} 09^{\prime \prime}$ & 190 \\
\hline 27. & Japra River, Gradine & 2013.07.28. & $44^{\circ} 53^{\prime} 39^{\prime \prime}$ & $16^{\circ} 25^{\prime} 47^{\prime \prime}$ & 185 \\
\hline 28. & Ovanjska River, Marini 2 & 2013.07.28. & $44^{\circ} 53^{\prime} 35^{\prime \prime}$ & $16^{\circ} 29^{\prime} 09^{\prime \prime}$ & 219 \\
\hline 29. & Mliništa Stream, Johovica & 2013.07.28. & $44^{\circ} 53^{\prime} 33^{\prime \prime}$ & $16^{\circ} 22^{\prime} 14^{\prime \prime}$ & 221 \\
\hline 30. & Stream at Latinovića Dolina & 2013.07.28. & $44^{\circ} 53^{\prime} 28^{\prime \prime}$ & $16^{\circ} 17^{\prime} 31^{\prime \prime}$ & 196 \\
\hline 31. & Spring and stream at Zelenac & 2013.07.28. & $44^{\circ} 53^{\prime} 25^{\prime \prime}$ & $16^{\circ} 17^{\prime} 44^{\prime \prime}$ & 194 \\
\hline 32. & Bosanska Krupa, city & 2013.07.28. & $44^{\circ} 53^{\prime} 01^{\prime \prime}$ & $16^{\circ} 09^{\prime} 15^{\prime \prime}$ & 155 \\
\hline 33. & Una River at Kostela, Bihać & 2005.11.03. & $44^{\circ} 52^{\prime} 56^{\prime \prime}$ & $15^{\circ} 54^{\prime} 00^{\prime \prime}$ & 215 \\
\hline 34. & Stream near Mrkalji & 2013.07.28. & $44^{\circ} 52^{\prime} 53^{\prime \prime}$ & $16^{\circ} 23^{\prime} 45^{\prime \prime}$ & 209 \\
\hline 35. & Dobrovac Stream & 2013.07.27. & $44^{\circ} 52^{\prime} 27^{\prime \prime}$ & $16^{\circ} 10^{\prime} 27^{\prime \prime}$ & 174 \\
\hline 36. & Stream at Izačić Kula & 2013.07.26. & $44^{\circ} 52^{\prime} 23^{\prime \prime}$ & $15^{\circ} 46^{\prime} 41^{\prime \prime}$ & 280 \\
\hline 37. & $\begin{array}{l}\text { Canyon of the Una River, near Bosanska } \\
\text { Krupa }\end{array}$ & 2012.08.20. & $44^{\circ} 52^{\prime} 00^{\prime \prime}$ & $16^{\circ} 05^{\prime} 42^{\prime \prime}$ & 242 \\
\hline
\end{tabular}




\begin{tabular}{|c|c|c|c|c|c|}
\hline 38. & Small stream at Papari & 2013.07.26. & $44^{\circ} 51^{\prime} 15^{\prime \prime}$ & $15^{\circ} 47^{\prime} 52^{\prime \prime}$ & 231 \\
\hline 39. & Pond at Kamenica, Bihać & 2012.08.20. & $44^{\circ} 50^{\prime} 10^{\prime \prime}$ & $15^{\circ} 49^{\prime} 55^{\prime \prime}$ & 215 \\
\hline 40. & Mrežnica Stream, Klokot & 2013.07.26. & $44^{\circ} 49^{\prime} 44^{\prime \prime}$ & $15^{\circ} 49^{\prime} 25^{\prime \prime}$ & 215 \\
\hline 41. & Hatiraj Stream, Čirkići & 2013.07.28. & $44^{\circ} 49^{\prime} 32^{\prime \prime}$ & $16^{\circ} 30^{\prime} 50^{\prime \prime}$ & 354 \\
\hline 42. & Klokot spring and River & 2013.07.26. & $44^{\circ} 49^{\prime} 24^{\prime \prime}$ & $15^{\circ} 48^{\prime} 18^{\prime \prime}$ & 220 \\
\hline 43. & Bihać, city & 2013.07.26. & $44^{\circ} 48^{\prime} 51^{\prime \prime}$ & $15^{\circ} 52^{\prime} 34^{\prime \prime}$ & 224 \\
\hline 44. & Ponds at Vedro Polje & 2012.08.19. & $44^{\circ} 48^{\prime} 39^{\prime \prime}$ & $15^{\circ} 50^{\prime} 19^{\prime \prime}$ & 220 \\
\hline 45. & Lisa rivulet, Vedro Polje & 2012.08.19. & $44^{\circ} 48^{\prime} 23^{\prime \prime}$ & $15^{\circ} 49^{\prime} 24^{\prime \prime}$ & 213 \\
\hline 46. & Dry stream at Rastići polje & 2013.07.26. & $44^{\circ} 48^{\prime} 00^{\prime \prime}$ & $15^{\circ} 50^{\prime} 34^{\prime \prime}$ & 240 \\
\hline 47. & Suvajčina Stream, Gornje Suvaje & 2013.07.28. & $44^{\circ} 46^{\prime} 55^{\prime \prime}$ & $16^{\circ} 09^{\prime} 27^{\prime \prime}$ & 416 \\
\hline 48. & Banovac Spring, Naprelje & 2013.07.28. & $44^{\circ} 46^{\prime} 49^{\prime \prime}$ & $16^{\circ} 30^{\prime} 00^{\prime \prime}$ & 374 \\
\hline 49. & Suvajčina Stream, Gornje Suvaje 2 & 2013.07.28. & $44^{\circ} 46^{\prime} 30^{\prime \prime}$ & $16^{\circ} 09^{\prime} 21^{\prime \prime}$ & 444 \\
\hline 50. & Una River, Ripač & 2012.08.19. & $44^{\circ} 46^{\prime} 20^{\prime \prime}$ & $15^{\circ} 56^{\prime} 51^{\prime \prime}$ & 228 \\
\hline 51. & Zavaljska Draga Stream, Zavalje & 2013.07.26. & $44^{\circ} 46^{\prime} 19^{\prime \prime}$ & $15^{\circ} 50^{\prime} 06^{\prime \prime}$ & 438 \\
\hline 52. & Dobrenica Stream, Dobrenica & 2013.07.29. & $44^{\circ} 45^{\prime} 58^{\prime \prime}$ & $15^{\circ} 53^{\prime} 39^{\prime \prime}$ & 273 \\
\hline 53. & Skočajska Draga Stream near Crno vrelo & 2013.07.26. & $44^{\circ} 45^{\prime} 35^{\prime \prime}$ & $15^{\circ} 50^{\prime} 35^{\prime \prime}$ & 452 \\
\hline 54. & Stream at Kuline, Lušci polje & 2013.07.28. & $44^{\circ} 45^{\prime} 24^{\prime \prime}$ & $16^{\circ} 26^{\prime} 30^{\prime \prime}$ & 397 \\
\hline 55. & $\begin{array}{l}\text { Ponds and stream between Lušci Palanka } \\
\text { and Šiprage }\end{array}$ & 2012.06.26. & $44^{\circ} 44^{\prime} 21^{\prime \prime}$ & $16^{\circ} 27^{\prime} 03^{\prime \prime}$ & 383 \\
\hline 56. & Praštali, by the road, Lušci polje & 2012.06.26. & $44^{\circ} 43^{\prime} 00^{\prime \prime}$ & $16^{\circ} 26^{\prime} 41^{\prime \prime}$ & 382 \\
\hline 57. & Oko Spring, Lušci polje & $\begin{array}{l}\text { (a) 2012.06.26., } \\
\text { (b) 2014.08.04., } \\
\text { (c) 2014.08.05. }\end{array}$ & $44^{\circ} 42^{\prime} 09^{\prime \prime}$ & $16^{\circ} 27^{\prime} 46^{\prime \prime}$ & 387 \\
\hline 58. & Ristinovac Spring, Lipa & 2013.07.29. & $44^{\circ} 41^{\prime} 40^{\prime \prime}$ & $16^{\circ} 04^{\prime} 33^{\prime \prime}$ & 720 \\
\hline 59. & $\begin{array}{l}\text { Madžarac Stream and nearby ponds, } \\
\text { Bjelaj, Bjelajsko polje }\end{array}$ & $\begin{array}{l}\text { (a) 2012.06.27., } \\
\text { (b) 2012.08.19. }\end{array}$ & $44^{\circ} 34^{\prime} 55^{\prime \prime}$ & $16^{\circ} 11^{\prime} 29^{\prime \prime}$ & 655 \\
\hline 60. & $\begin{array}{l}\text { Pećina Pond, Donji Rašinovac, Bosanski } \\
\text { Petrovac }\end{array}$ & 2012.06.27. & $44^{\circ} 33^{\prime} 44^{\prime \prime}$ & $16^{\circ} 21^{\prime} 01^{\prime \prime}$ & 658 \\
\hline 61. & $\begin{array}{l}\text { Small canal and stream at Revenik, } \\
\text { Petrovačko polje }\end{array}$ & 2012.06.27. & $44^{\circ} 32^{\prime} 32^{\prime \prime}$ & $16^{\circ} 20^{\prime} 28^{\prime \prime}$ & 632 \\
\hline 62. & Stream at Bukovača, Petrovačko polje & 2012.06.27. & $44^{\circ} 30^{\prime} 38^{\prime \prime}$ & $16^{\circ} 25^{\prime} 48^{\prime \prime}$ & 700 \\
\hline 63. & Una River at Martinbrod & $\begin{array}{l}\text { (a) 2009.08.11., } \\
\text { (b) 2010.09.05. }\end{array}$ & $44^{\circ} 28^{\prime} 53^{\prime \prime}$ & $16^{\circ} 08^{\prime} 34^{\prime \prime}$ & 333 \\
\hline 64. & Near the road to Martinbrod & 2010.09 .05 . & $44^{\circ} 27^{\prime} 11^{\prime \prime}$ & $16^{\circ} 15^{\prime} 56^{\prime \prime}$ & 652 \\
\hline 65. & Bastasi Fishpond & 2010.09 .05$. & $44^{\circ} 24^{\prime} 10^{\prime \prime}$ & $16^{\circ} 18^{\prime} 53^{\prime \prime}$ & 450 \\
\hline 66. & Bastašica Spring & 2010.09.05. & $44^{\circ} 23^{\prime} 39^{\prime \prime}$ & $16^{\circ} 19^{\prime} 36^{\prime \prime}$ & 460 \\
\hline 67. & Unac River, Drvar & 2005.11.03. & $44^{\circ} 22^{\prime} 52^{\prime \prime}$ & $16^{\circ} 23^{\prime} 00^{\prime \prime}$ & 466 \\
\hline 68. & Unac River, near cave, Drvar & 2010.09.05. & $44^{\circ} 22^{\prime} 48^{\prime \prime}$ & $16^{\circ} 23^{\prime} 14^{\prime \prime}$ & 471 \\
\hline 69. & By the road between Petrovac and Drvar & 2013.07.25. & $44^{\circ} 20^{\prime} 56^{\prime \prime}$ & $16^{\circ} 21^{\prime} 37^{\prime \prime}$ & 692 \\
\hline 70. & Ljeskovica Stream, Mrđe & 2013.07.25. & $44^{\circ} 19^{\prime} 04^{\prime \prime}$ & $16^{\circ} 29^{\prime} 54^{\prime \prime}$ & 560 \\
\hline 71. & Joševac Stream, Jovandići & 2013.07.25. & $44^{\circ} 13^{\prime} 30^{\prime \prime}$ & $16^{\circ} 19^{\prime} 57^{\prime \prime}$ & 814 \\
\hline 72. & Pečenačko Lake & 2013.07.25. & $44^{\circ} 11^{\prime} 15^{\prime \prime}$ & $16^{\circ} 21^{\prime} 03^{\prime \prime}$ & 822 \\
\hline 73. & Korana River, Veliki Obalj & 2013.07.25. & $44^{\circ} 10^{\prime} 36^{\prime \prime}$ & $16^{\circ} 23^{\prime} 05^{\prime \prime}$ & 798 \\
\hline 74. & Pond near Veliki Obalj & 2013.07.25. & $44^{\circ} 10^{\prime} 28^{\prime \prime}$ & $16^{\circ} 24^{\prime} 39^{\prime \prime}$ & 797 \\
\hline 75. & Korana River at Pašića polje & 2013.07.25. & $44^{\circ} 09^{\prime} 45^{\prime \prime}$ & $16^{\circ} 23^{\prime} 03^{\prime \prime}$ & 793 \\
\hline
\end{tabular}


in the area of Drvar and Bosansko Grahovo belong to Canton 10 (Livno Canton), while several localities $(11,19,21,22,24,27-31,34)$ situated south of Bosanski Novi are in the territory of Republika Srpska (AnONYmous, 2012).

\section{Data collection}

The Odonata fauna of the northwestern part of Bosnia was investigated during two short trips in 2012 (26 - 27 June and 19 - 20 August) and one longer trip in 2013 (25 - 29 July). In addition, all known publications, the collections of the National Museum of Bosnia and Herzegovina as well as the dragonfly database of Bosnia and Herzegovina, were checked for records referring to the study region. During this study, mainly adults were investigated. Adults were mainly visually observed or caught with a hand net and identified in the field. Several voucher specimens were collected and deposited in the entomological collections of the National Museum of Bosnia and Herzegovina. Larvae and exuviae were searched for only sporadically. They were collected and identified in the laboratory.

Records were gathered from 75 localities distributed between $120 \mathrm{~m}$ a.s.l. and $822 \mathrm{~m}$ a.s.l. (Tab. 1., Fig. 1.).

\section{RESULTS}

Altogether, 275 odonate records of 36 species from 75 different localities were collected (Tab. 2). Both hitherto known species for the region were found during this study.

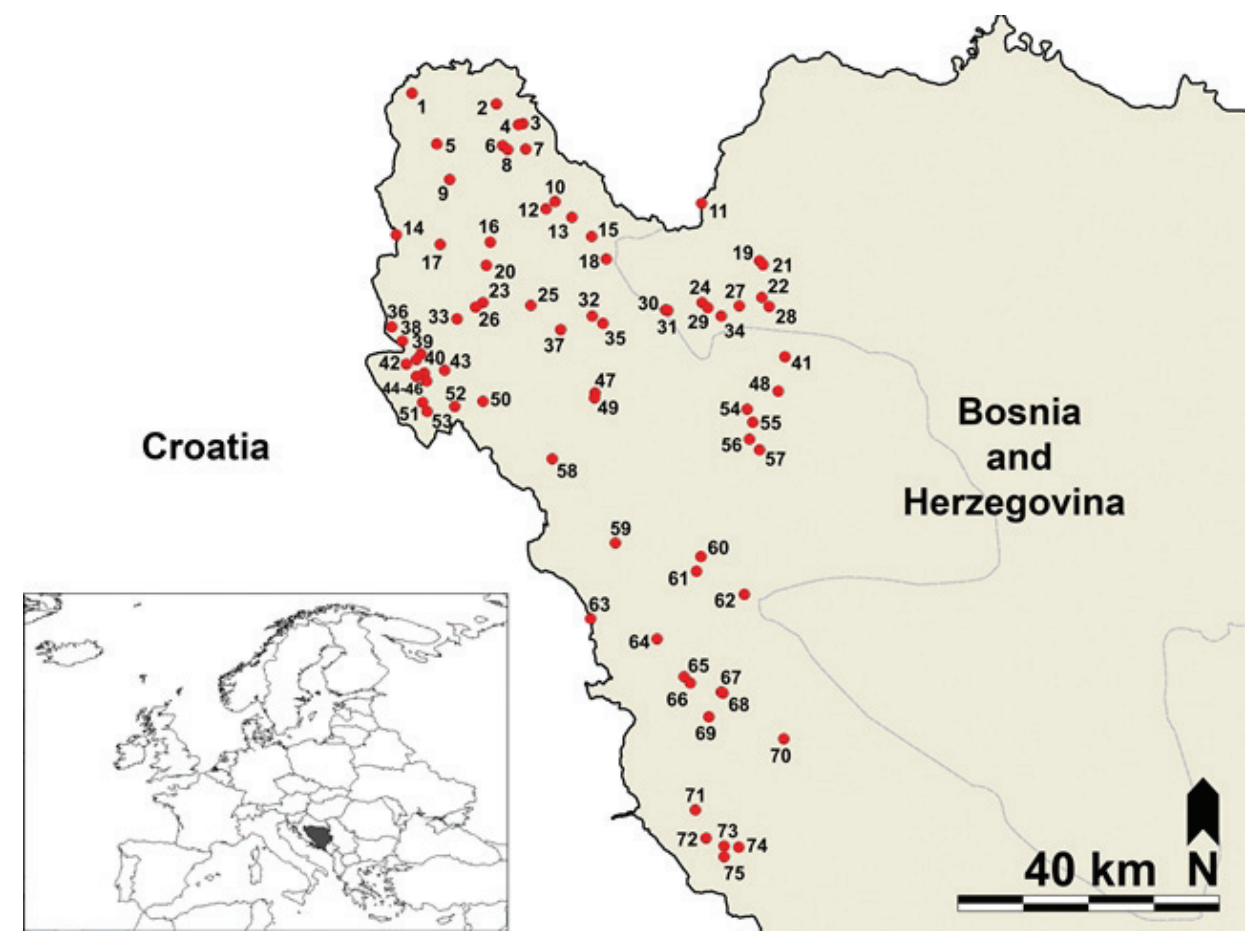

Fig. 1. Geographical position of the study area with the locations of dragonfly records. The numeration of each locality is presented in Tab. 1. 
Tab. 2. List of species recorded in northwestern Bosnia with reference to the specific locality $(*=$ marks two species previously known from the region).

\begin{tabular}{|c|c|c|}
\hline No. & Species name & Locality \\
\hline \multicolumn{3}{|c|}{ CALOPTERYGIDAE } \\
\hline 1. & Calopteryx splendens (Harris, 1782) & $\begin{array}{l}2,5,6,7,8,11,15,23,35,40,42,52,57(\mathrm{~b})(\mathrm{c}) \\
61,63(\mathrm{a}), 73\end{array}$ \\
\hline 2. & Calopteryx virgo (Linnaeus, 1758) * & $\begin{array}{l}4,5,6,7,9,10,11,12,13,14,15,16,17,19,20 \\
21,22,23,24,27,28,29,30,31,32,33,34,35 \\
36,38,40,41,42,46,48,51,52,53,54,55 \\
57(\mathrm{a}), 61,62,43,70,71,73\end{array}$ \\
\hline \multicolumn{3}{|c|}{ LESTIDAE } \\
\hline 3. & Lestes dryas Kirby, 1890 & 74,75 \\
\hline 4. & Lestes barbarus (Fabricius, 1798) & $44,65,72,74,75$ \\
\hline 5. & Lestes virens (Charpentier, 1825) & $17,46,72,75$ \\
\hline 6. & Chalcolestes viridis (Vander Linden, 1825) & 44 \\
\hline 7. & Chalcolestes parvidens (Artobolevskii, 1929) & 16 \\
\hline \multicolumn{3}{|c|}{ COENAGRIONIDAE } \\
\hline 8. & Ischnura elegans (Vander Linden, 1820) & $7,16,17,39,40,45,59(a)(b), 60,65,73$ \\
\hline 9. & Ischnura pumilio (Charpentier, 1825) & $39,46,59(a)(b), 60,61$ \\
\hline 10. & Enallagma cyathigerum (Charpentier, 1840) & 7, 16, 59(a), 60, 72 \\
\hline 11. & Coenagrion puella (Linnaeus, 1758) & $7,16,48,50,58,59(a), 60,72,73,75$ \\
\hline 12. & Coenagrion ornatum (Selys, 1850) & 59(a), 61 \\
\hline 13. & Coenagrion scitulum (Rambur, 1842) & $59(b), 60,61,75$ \\
\hline 14. & Erythromma lindenii (Selys, 1840) & $2,7,16,39,40,75$ \\
\hline 15. & Pyrrhosoma nymphula (Sulzer, 1776) & 73 \\
\hline \multicolumn{3}{|c|}{ PLATYCNEMIDIDAE } \\
\hline 16. & Platycnemis pennipes (Pallas, 1771) & $\begin{array}{l}2,5,6,7,8,11,16,17,23,37,40,42,48,60,61 \\
73\end{array}$ \\
\hline \multicolumn{3}{|c|}{ AESHNIDAE } \\
\hline 17. & Aeshna mixta Latreille, 1805 & $25,41,42,47,49,52,53,58,63(\mathrm{~b}), 64,65,66,68$ \\
\hline 18. & Aeshna affinis Vander Linden, 1820 & $44,46,69,72,74,75$ \\
\hline 19. & Aeshna cyanea (Müller, 1764) & 16,58 \\
\hline 20. & Anax imperator Leach, 1815 & $6,7,16,42,60$ \\
\hline \multicolumn{3}{|c|}{ GOMPHIDAE } \\
\hline 21. & Gomphus vulgatissimus (Linnaeus, 1758) & 6 \\
\hline 22. & Onychogomphus forcipatus (Linnaeus, 1758) * & $\begin{array}{l}\text { 1, 2, 6, 10, 15, 16, 18(a)(b), 19, 27, 28, 29, 30, 35, } \\
37,40,56,57(b), 67\end{array}$ \\
\hline \multicolumn{3}{|c|}{ CORDULEGASTERIDAE } \\
\hline 23. & Cordulegaster heros Theischinger, 1979 & $3,4,13,15,21,35,51,53$ \\
\hline 24. & Cordulegaster bidentata Selys, 1843 & 26,53 \\
\hline \multicolumn{3}{|c|}{ CORDULIIDAE } \\
\hline 25. & Somatochlora meridionalis Nielsen, 1935 & $\begin{array}{l}3,4,5,6,7,16,17,21,28,31,35,40,41,42,46 \\
47,52,53\end{array}$ \\
\hline 26. & Somatochlora flavomaculata (Vander Linden, 1825) & $5,17,45,73$ \\
\hline \multicolumn{3}{|c|}{ LIBELLULIDAE } \\
\hline 27. & Libellula depressa Linnaeus, 1758 & $7,16,22,40,42,46,59(a)(b), 60,61,74$ \\
\hline
\end{tabular}




\begin{tabular}{|l|l|l|}
\hline 28. & Libellula fulva (Müller, 1764) & 60 \\
\hline 29. & Orthetrum cancellatum (Linnaeus, 1758) & 65,75 \\
\hline 30. & Orthetrum albistylum (Selys, 1848) & 7,39 \\
\hline 31. & Orthetrum coerulescens (Fabricius, 1798) & $7,12,17,22,28,31,34,45,59(\mathrm{a})(\mathrm{b})$ \\
\hline 32. & Orthetrum brunneum (Fonscolombe, 1837) & $7,39,40,46,52,59(\mathrm{a})(\mathrm{b}), 61$ \\
\hline 33. & Sympetrum sanguineum (Müller, 1764) & $\begin{array}{l}2,5,7,8,17,31,39,40,42,44,45,46,48,52,65, \\
72,73,74,75\end{array}$ \\
\hline 34. & Sympetrum flaveolum (Linnaeus, 1758) & 73,75 \\
\hline 35. & Sympetrum striolatum (Charpentier, 1840) & $16,59(\mathrm{~b}), 75$ \\
\hline 36. & Crocothemis erythraea (Brullé, 1832) & 7 \\
\hline
\end{tabular}

Most important was the discovery of two species mentioned in Annex II of the Habitats Directive, Coenagrion ornatum (Selys, 1850) and Cordulegaster heros Theischinger, 1979, together with records of two species rare in B\&H, Chalcolestes viridis (Vander Linden, 1825) and Coenagrion scitulum (Rambur, 1842) (Kulijer et al., 2013, 2016).

The majority of the observed species are widespread in the country (Kulijer et al., 2013). The most recorded were: Calopteryx virgo (Linnaeus, 1758) (48 localities), Sympetrum sanguineum (Müller, 1764) (20), Onychogomphus forcipatus (Linnaeus, 1758) (19), Somatochlora meridionalis Nielsen, 1935 (18), Calopteryx splendens (Harris, 1782) (17), Platycnemis pennipes (Pallas, 1771) (16). The dragonfly-richest sites were L. 7 - lake near mine at Bajraktarevići (15 species) and L. 16 - stream and small lake at Donja Koprivna (13 species). Both sites are characterized by a combination of flowing and stagnant water with rich and diverse vegetation.

\section{Systematic account}

Abbreviations used: M - male(s), F - female(s), MA - mature adult(s), Juv/Ten juvenile(s)/teneral(s), Lar - larva(e), Ex - exuvium(ae), Cop - copula(e)/tandem(s), Ovip - ovipositing.

\section{CALOPTERYGIDAE}

\section{Calopteryx splendens (Harris, 1782)}

Loc. 2: >10MA; Loc. 5: 3M, 1F; Loc. 6: >10MA; Loc. 7: 2M; Loc. 8: 100MA; Loc. 11: 3M; Loc. 15: >10MA; Loc. 23: 3M, 1F; Loc. 35: 1M, 1F; Loc. 40: 15MA; Loc. 42: 30MA; Loc. 52: <10MA; Loc. 57(b): 1F, (c): 1F; Loc. 61: 15M, 5F; Loc. 63(a): 1M; Loc. 73: 30MA

\section{Calopteryx virgo (Linnaeus, 1758)}

Loc. 4: >100MA; Loc. 5: 2F; Loc. 6: <10MA; Loc. 7: 2M, 2F; Loc. 9: 2M, 4F; Loc. 10: >10MA; Loc. 11: 1M; Loc. 12: 1M, 1F; Loc. 13: >10MA; Loc. 14: 7MA; Loc. 15: >100MA; Loc. 16: 3M, 3F; Loc. 17: 3M, 2F; Loc. 19: 2M, 2F; Loc. 20: 30MA; Loc. 21: 50MA; Loc. 22: 2M, 2F; Loc. 23: 3M, 1Ovip; Loc. 24: >10MA; Loc. 27: >10MA; Loc. 28: >10MA; Loc. 29: >10MA; Loc. 30: >10MA; Loc. 31: >10MA; Loc. 32: 1M; Loc. 33: 1MA; Loc. 34: 1M; Loc. 35: >10MA; Loc. 36: 10M, 4F; Loc. 38: 7M, 2F; Loc. 40: 20MA; Loc. 41: >10MA; Loc. 42: 30MA; Loc. 43: 1F; Loc. 46: 8M, 3F; Loc. 48: >10MA; Loc. 51: 10M, 5F; Loc. 52: <10MA; Loc. 53: <10MA; Loc. 54: 3M; Loc. 55: >10MA; Loc. 57(a): 1F; Loc. 61: 2F; Loc. 62: 20M, 4F; Loc. 70: 10M; Loc. 71: 1M; Loc. 73: 20MA 


\section{LESTIDAE}

Lestes dryas Kirby, 1890

Loc. 74: >10MA; Loc. 75: 15MA

Lestes barbarus (Fabricius, 1798)

Loc. 44: 1F; Loc. 65: 1F; Loc. 72: 1MA; Loc. 74: >10MA, >10Cop; Loc. 75: 2MA

Lestes virens (Charpentier, 1825)

Loc. 17: 1F; Loc. 46: 1M; Loc. 72: 1MA; Loc. 75: 5MA

Chalcolestes viridis (Vander Linden, 1825)

Loc. 44: 2M, 1Cop

Chalcolestes parvidens (Artobolevskii, 1929)

Loc. 16: 3 Juv/Ten

\section{COENAGRIONIDAE}

\section{Ischnura elegans (Vander Linden, 1820)}

Loc. 7: <10MA; Loc. 16: <10MA; Loc. 17: 1M; Loc. 39: >10MA, 1Cop; Loc. 40: 2MA; Loc. 45: 1M; Loc. 59(a): 1M, (b): 2M; Loc. 60: >10MA, 1Cop; Loc. 65: 6M, 1F, 2Cop; Loc. 73: $2 \mathrm{M}$

\section{Ischnura pumilio (Charpentier, 1825)}

Loc. 39: 5MA; Loc. 46: 1Cop; Loc. 59(a): 15M, 2Cop, (b): >10MA,<10Cop; Loc. 60: $<10 \mathrm{MA}$; Loc. 61: 10M, 1F, 1Cop

\section{Enallagma cyathigerum (Charpentier, 1840)}

Loc. 7: <10MA; Loc. 16: >10MA, <10Cop; Loc. 59(a): 1Cop; Loc. 60: >10MA; Loc. 72: $5 \mathrm{M}, 2 \mathrm{Cop}$

\section{Coenagrion puella (Linnaeus, 1758)}

Loc. 7: <10M; Loc. 16: >10MA; Loc. 48: 2M; Loc. 50: 10M, 1F; Loc. 58: >10M; Loc. 59(a): $>10 \mathrm{MA},<10$ Cop; Loc. 60: >10MA, <10Cop, <10Ovip; Loc. 72: 2M; Loc. 73: >10MA, <10Cop, $<10$ Ovip; Loc. 75: 10M, 2Cop

\section{Coenagrion ornatum (Selys, 1850)}

Loc. 59(a): 30M, 3F, 3Cop; Loc. 61: 3M

\section{Coenagrion scitulum (Rambur, 1842)}

Loc. 59(b): 1M; Loc. 60: 2M, 2Cop; Loc. 61: 1F; Loc. 75: 1M, 2Cop

\section{Erythromma lindenii (Selys, 1840)}

Loc. 2: >10MA; Loc. 7: >100MA, >10Cop, <10Ovip; Loc. 16: >100MA, >10Cop, >10Ovip; Loc. 39: 1M; Loc. 40: 20M; Loc. 75: 50M, 2Cop

\section{Pyrrhosoma nymphula (Sulzer, 1776)}




\section{PLATYCNEMIDIDAE}

Platycnemis pennipes (Pallas, 1771)

Loc. 2: >10MA, <10Cop; Loc. 5: >100MA, >10Juv/Ten; Loc. 6: >10MA, <10Cop; Loc. 7: $>$ 10MA, <10Cop, <10Ovip; Loc. 8: >10MA; Loc. 11: 2M, 2F; Loc. 16: >10MA, <10Cop; Loc. 17: 3M; Loc. 23: 1M; Loc. 37: 1M, 2F; Loc. 40: >10MA,>10Cop; Loc. 42: 20MA,1Cop; Loc. 48: <10MA, <10Cop; Loc. 60: 1Cop; Loc. 61: 1M, 1F; Loc. 73: >10MA, <10Cop, <10Ovip, $<10$ Juv/Ten

\section{AESHNIDAE}

\section{Aeshna mixta Latreille, 1805}

Loc. 25: <10MA; Loc. 41: 1MA; Loc. 42: 1M; Loc. 47: <10MA; Loc. 49: 1MA; Loc. 52: 2MA; Loc. 53: 1MA; Loc. 58: 1MA; Loc. 63(b): 4M; Loc. 64: 3M, 2F; Loc. 65: 2M; Loc. 66: 2M, 1F; Loc. 68: 1F

\section{Aeshna affinis Vander Linden, 1820}

Loc. 44: 3M; Loc. 46: 1M; Loc. 69: <10MA; Loc. 72: 1M; Loc. 74: 1M; Loc. 75: 5M, 1Cop Aeshna cyanea (Müller, 1764)

Loc. 16: 1M; Loc. 58: $1 \mathrm{~F}$

\section{Anax imperator Leach, 1815}

Loc. 6: 2M; Loc. 7: 3M; Loc. 16: 4M, 2F, 2Ovip; Loc. 42: 1M; Loc. 60: 2M, 2F, 1Ovip

\section{GOMPHIDAE}

\section{Gomphus vulgatissimus (Linnaeus, 1758)}

Loc. 6: $2 \mathrm{M}$

\section{Onychogomphus forcipatus (Linnaeus, 1758)}

Loc. 1: 7Lar; Loc. 2: >10MA; Loc. 6: 2M; Loc. 10: 1M; Loc. 15: 3M; Loc. 16: 4M; Loc. 18(a): 2Lar, (b): 2Lar; Loc. 19: 2M; Loc. 27: 5M; Loc. 28: 1M; Loc. 29: 1M; Loc. 30: 2M; Loc. 35: 1M; Loc. 37: 1F; Loc. 40: 1F; Loc. 56: 1M; Loc. 57(b): 1M; Loc. 67: 1Lar

\section{CORDULEGASTERIDAE}

\section{Cordulegaster heros Theischinger, 1979}

Loc. 3: 1M; Loc. 4: 1M; Loc. 13: 3M; Loc. 15: 3M; Loc. 21: 1M; Loc. 35: 1M; Loc. 51: 3M; Loc. 53: $15 \mathrm{M}$

\section{Cordulegaster bidentata Selys, 1843}

Loc. 26: 1M; Loc. 53: 10M

\section{CORDULIIDAE}

\section{Somatochlora meridionalis Nielsen, 1935}

Loc. 3: 1MA; Loc. 4: 1MA; Loc. 5: 1M; Loc. 6: 1MA; Loc. 7: 1M; Loc. 16: 100MA; Loc. 17: 1MA; Loc. 21: 4M; Loc. 28: 1M; Loc. 31: >10MA; Loc. 35: 1MA; Loc. 40: 2M; Loc. 41: 1M; Loc. 42: 1MA; Loc. 46: 2M; Loc. 47: 1M; Loc. 52: <10MA; Loc. 53: 1MA 


\section{Somatochlora flavomaculata (Vander Linden, 1825)}

Loc. 5: 1M; Loc. 17: 1M; Loc. 45: 1M; Loc. 73: 1M

\section{LIBELLULIDAE}

\section{Libellula depressa Linnaeus, 1758}

Loc. 7: 1M; Loc. 16: 3M, 1F; Loc. 22: 1M; Loc. 40: 1F; Loc. 42: 1F; Loc. 46: 1M; Loc. 59(a): 6M, (b): 1F; Loc. 60: 6M, 1Cop; Loc. 61: 3M; Loc. 74: 2M

Libellula fulva (Müller, 1764)

Loc. 60: $2 \mathrm{M}$

Orthetrum cancellatum (Linnaeus, 1758)

Loc. 65: 1M; Loc. 75: 2M

Orthetrum albistylum (Selys, 1848)

Loc. 7: >10MA, 1Ovip; Loc. 39: 1M

\section{Orthetrum coerulescens (Fabricius, 1798)}

Loc. 7: 5M; Loc. 12: 1M; Loc. 17:1M, 1F; Loc. 22: 2M, 1F; Loc. 28: 1M, 1F; Loc. 31: 30M, 2F, 1MA; Loc. 34: 1M; Loc. 45: 2M; Loc. 59(a): 3M, 1Juv, (b): 40MA, 10Cop, 5Ovip

\section{Orthetrum brunneum (Fonscolombe, 1837)}

Loc. 7: 10M, 1F; Loc. 39: 2M; Loc. 40: 20M, 2F, 1Cop, 2Ovip; Loc. 46: 7M; Loc. 52: 3M; Loc. 59(a): 15M, 2Cop, (b): 3M, 1Ovip; Loc. 61: 1M

\section{Sympetrum sanguineum (Müller, 1764)}

Loc. 2: 2M; Loc. 5: 1F; Loc. 7: 5M, 1F, 2Cop; Loc. 8: 1M; Loc. 17: 2M, 1F; Loc. 31: 1M; Loc. 39: 1M; Loc. 40: 15M, 1F; Loc. 42: 1M; Loc. 44: 6M; Loc. 45: 2M; Loc. 46: 3M; Loc. 48: 1M; Loc. 52: 1F; Loc. 65: 3M; Loc. 72: 3M, 1F; Loc. 73: 5M, 1Ovip; Loc. 74: 2M; Loc. 75: 10M, 10Cop, 3Ovip

\section{Sympetrum flaveolum (Linnaeus, 1758)}

Loc. 73: 2M; Loc. 75: 1M

\section{Sympetrum striolatum (Charpentier, 1840)}

Loc. 16: 2Juv/Ten; Loc. 59(b): 5M, 5Juv/Ten, <10Ex; Loc. 75: 3Juv/Ten

\section{Crocothemis erythraea (Brullé, 1832)}

Loc. 7: $2 \mathrm{M}$

\section{DISCUSSION}

Regional studies are of major importance in providing missing pieces of the distributional puzzle, and essential for an efficient protection of threatened species. Although this was a time-restricted survey, the number of 36 species recorded during the research is quite high, representing $56 \%$ of all dragonfly species of the country (Kulijer et al., 2013; Kulijer \& Miljević, 2015).

Among the most widely distributed species in the study area, the majority inhabit lotic waters (e.g. C. virgo, C. splendens, P. pennipes, O. forcipatus, S. meridionalis) while only 
S. sanguineum prefers lentic habitats (DiJKSTRA \& LEwington, 2006). As most of the investigated localities were brooks, streams or small rivers, the high dominance of rheophilic species is not surprising. Two Calopteryx spp. can be found in Bosnia and Herzegovina, C. splendens and C. virgo. Both are typical of running waters, common in the country and often found syntopically. In comparison with C. splendens, C. virgo prefers smaller, colder and shaded flowing waters (DIJKSTRA \& LEwINGTON, 2006). It was found at almost all the investigated brooks, streams and small rivers in the study area. The eurytopic $P$. pennipes is one of the most widespread dragonfly species in the country (Kulijer et al., 2013), and this was reflected in the study area. In accordance with the habitat preferences of O. forcipatus (DijKsTRA \& LEWINGTON, 2006), many of the rocky bottom streams and rivers inspected during the study were found to be suitable habitat type for the species. Somatochlora meridionalis inhabits small and shaded streams and brooks (DIJKSTRA \& Lewington, 2006), another abundant habitat type in the region. Although it typically reproduces in stagnant water bodies, $S$. sanguineum can be often seen far from suitable habitats (DijKstra \& Lewington, 2006). In the study area, it was observed at different habitat types including meadows, fields and along roads and rivers. It is the most abundant Sympetrum spp. and one of the most frequently observed dragonflies in B\&H (KULIJER et al., 2013).

During the study, six species were recorded at only one locality, namely Chalcolestes parvidens (Artobolevskii, 1929), C. viridis, Pyrrhosoma nymphula (Sulzer, 1776), Gomphus vulgatissimus (Linnaeus,1758), Libellula fulva (Müller, 1764) and Crocothemis erythraea (Brullé, 1832). Although P. nymphula, G. vulgatissimus and L. fulva are common in B\&H, they are typical spring species that are quite rare during the late summer, when most of the study was conducted (Dijkstra \& Lewington, 2006; Kulijer et al., 2012, 2013). Additionally, L. fulva inhabits larger freshwater habitats with slow water currents and rich riparian vegetation (DiJKsTRA \& LEWINGTON, 2006). The scarcity of such habitats in the study area combined with the late-summer timing of the study resulted in a low occurrence of this species. Crocothemis erythraea is a common species countrywide (Kulijer et al., 2013) with a preference for open stagnant water habitats (DIJKSTRA \& LEWINGTON, 2006). It was found only at a small lake near Barjaktarevići (L. 7), the characteristic habitat type for the species, but a rare habitat among the investigated sites. This was probably the main reason why an otherwise common species was observed only once.

The genus Chalcolestes Kennedy, 1920 is represented with two species: C. parvidens and C. viridis, whose range widely overlaps in the Balkans (Olias et al., 2007). Both species are present throughout the country with $C$. parvidens being more frequently recorded in the north and south, while C. viridis is more common in the mountainous regions of the Dinaric Alps (Kuljer et al., 2013, 2016). During the study, each species was recorded at a single locality. The record of C. viridis at Vedro polje near Bihać (L. 44) is more interesting as this species is much scarcer in the country than its congener. Several specimens, including one copula, were found in dense Salix sp. shrubs at several small, almost dry and muddy ponds. Typical macrophyte vegetation at these ponds was scarce, probably due to their mostly temporary character. The ponds seemed to be artificial and the area is currently abandoned and used as a waste disposal site. It is expected that both species are more common in the region, but further investigations are required in late summer and autumn to confirm this.

In Europe, C. scitulum is widespread in its southern part, where it is more common in the south-west than in the south-east. In the Balkans the species is widely present but its populations are scarce (Boudot \& Jović, 2015). This is also the case for the whole of 
Bosnia and Herzegovina, where data of this species are rare and scattered throughout the country (Kulijer et al., 2013, 2016). In Bosnia and Herzegovina C. scitulum prefers small, sunny and shallow standing waters, particularly ponds and small ditches in the Mediterranean and Alpine regions (Kulijer, 2012, 2014; Kulijer et al., 2013, 2016). In the study region, the species was found at several localities in its southern parts, in Pašića, Petrovačko and Bjelajsko polje (L 59, 60, 61, 75). At Donji Rašinovac (L. 60) it was recorded at a cattle pond, while at other localities the small temporary or permanent pools and ponds were associated with nearby streams or ditches. At the Korana River (L. 75) several individuals, including copulae were found in a concrete tank that apart from algae almost lacked any other vegetation. Lestes sponsa (Hansemann, 1823), Sympecma fusca (Vander Linden, 1820), Erythromma najas (Hansemann, 1823), Erythromma viridulum (Charpentier, 1840), Aeshna isoceles (Müller, 1767), Anax parthenope Selys, 1839, Brachytron pratense (Müller, 1764), Cordulia aenea (Linnaeus, 1758), Libellula quadrimaculata Linnaeus, 1758, Sympetrum fonscolombii (Selys, 1840) and Sympetrum meridionale (Selys, 1841) are some of the species that are common in neighboring regions of Bosnia and Herzegovina (Kulijer, 2012; Kulijer et al., 2013, 2016) and Croatia (Belančić et al., 2008; Franković \& Vilenica, 2009; Španić et al., 2012; Vilenica \& DijKstra, 2014; Vinko \& Vilenica, 2013), but were not found during this study. This could be primarily explained by the fact that these regions are better investigated, as this survey did not cover a full range of seasons and habitats. Among these are several species with an early flight season, such as $E$. najas, A. isoceles, A. parthenope, B. pratense, C. aenea and L. quadrimaculata, although $A$. isoceles and $A$. parthenope are also present during summer months. These species were probably missed due to the late period of the investigations, but also due to the scarcity of lentic habitat types.

The main reason why L. sponsa, S. fusca, E. viridulum, S. fonscolombii and S. meridionale were not recorded is the scarcity of stagnant water habitats at the localities surveyed. Species like L. sponsa, E. viridulum and S. meridionale are common summer species with a peak in July and August, while S. fusca and S. fonscolombii are present throughout the year as they have at least two generations in the region (DijKstra \& Lewington, 2006).

One of the largest stagnant water habitats in the region, Bastasi fishpond (L. 65), was investigated only partly in September when most species were not present anymore, so it should be revisited, both in spring and summer.

Further research, especially in the lower parts of the Una catchment that were not included in this study, could also result in new findings of several nationally rare species, i. e. E. najas, Aeshna grandis (Linnaeus, 1758), Gomphus flavipes (Charpentier, 1825), Epitheca bimaculata (Charpentier, 1825), Leucorrhinia pectoralis (Charpentier, 1825), Leucorrhinia caudalis (Charpentier, 1840) or Sympetrum vulgatum (Linnaeus, 1758). Some of them (E. najas and E. bimaculata) were recorded by Franković \& Vilenica (2009) at the lower reaches of the Una River in Croatia. These species are expected in the lowland region of north Bosnia, in habitats that are not present or are very rare in the investigated part of the Una River catchment. Species such as E. bimaculata, G. flavipes, L. pectoralis and L. caudalis are rare in the country. So far, they were recorded only in the northern lowland region along the Sava River and lower reaches of its main tributaries (KuLIJER et al., 2013; Kulijer \& Miljević, 2015). Generally, the conditions for these lowland species seem to be limited in the study area.

In Bosnia and Herzegovina A. grandis is a rare species found only at mountain lakes (e.g. Kuljuer et al., 2012, 2013; Kulijer \& Miljević, 2017), while in Croatia it also occurs in slow flowing rivers, oxbows and ponds/lakes in the lowlands and forests. Some of 
these habitats, such as Plitvice Lakes, are close to the border of this region (BELANčić et al., 2008). As similar habitats (slow flowing parts of the larger river in hilly areas with rich water vegetation) also exist along the middle part of the Una River in $\mathrm{B} \& \mathrm{H}$, there is a possibility that the species could be found here in the future. Jović et al. (2010) gave records of $A$. grandis from Mt. Šator, located several kilometers south of the study region. These individuals probably originate from mountain lakes and ponds in the vicinity, possibly Šatorsko or Glamočko Lake.

\section{Conservation reference}

The present study provided new observations of two species of European conservation concern, C. ornatum and C. heros. Both species are listed in the EU Habitats Directive, while C. heros is also listed as Vulnerable in the Mediterranean Red List of Dragonflies (De Knijf et al., 2015; KalKman et al., 2010; Riservato et al., 2009). The knowledge of their distribution is important for the establishment of the Natura 2000 network. In Bosnia and Herzegovina C. ornatum can mainly be found in May/June and it usually disappears by the beginning of July (Boudot \& Kulijer, 2015). Although most investigations were conducted later in the year, a brief inspection of several localities in karst poljes at the end of June resulted in two new localities and the first in the northwestern region. As the species is already known as regularly occurring in karst poljes of southwest Bosnia and west Herzegovina, this finding is not a surprise (Kulijer, 2012, 2014; Kulijer et al., 2013). In the present proposition of the potential Natura 2000 areas for $\mathrm{B} \& \mathrm{H}$, only one area for C. ornatum was listed. Cordulegaster heros was not even included in the country's reference list (Milanović et al., 2015).

Cordulegaster heros is a widespread species in Bosnia and Herzegovina, found at streams and small rivers particularly in the southern region, and it was also expected to be widely distributed in north Bosnia (Kulijer, 2012, 2014; Kulijer et al., 2012, 2013, 2016). The results of this study confirmed these expectations. Partially shaded brooks and streams in hilly areas, particularly in the northeastern part, were found to have stable populations of the species. Several of these sites are close to the Croatian border, such as two streams (L 3 and L 53) located only several km from the border. The results of this study suggest that some parts of the study area are particularly important for C. heros, a species whose habitats should receive special attention in the process of the designation of Natura 2000 areas in B\&H.

No Red List of the dragonflies of Bosnia and Herzegovina exists as yet. Although dragonflies are included in the Red Lists for both entities in Bosnia and Herzegovina, these lists do not reflect the actual threat status of dragonfly species in the entities, nor in the country. The Red List of Protected Species of Flora and Fauna of Republika Srpska („Crvena lista zaštićenih vrsta flore i faune Republike Srpske”) includes 46 dragonfly species, but does not provide any threat category for any of the species (SLUžBENI GLASNIK Republike SRPSKe, 2012). The list includes practically all the most common species (e.g. C. splendens, Libellula depressa Linnaeus, 1758, Ischnura elegans (Vander Linden, 1820)), but failed to include several species that are rare and/or threatened in Republika Srpska and Bosnia and Herzegovina, such as Caliaeschna microstigma (Schneider, 1845), E. bimaculata or S. vulgatum. All recorded species, apart from Somatochlora flavomaculata (Vander Linden, 1825), C. heros, Cordulegaster bidentata Selys, 1843 and L. fulva, are included in the Red List.

On the other hand, the recently published Red List of wild species and subspecies of plants, animals and fungi („Crvena lista divljih vrsta i podvrsta biljaka, životinja i gljiva”) 
in the Federation of Bosnia and Herzegovina does not assess any of the dragonfly species occurring in FB\&H to be endangered (Službeni list Federacije Bosne i Hercegovi$\mathrm{NE}, 2014$ ). Out of only three species mentioned on the list (all regarded as Near Threatened), Lestes barbarus (Fabricius, 1798) and C. heros were found during the survey.

This study contributes markedly to a better knowledge of the dragonfly fauna of the Una River catchment, an important area for biodiversity shared between Bosnia and Herzegovina and Croatia. Considering the size of the study area and the habitats diversity, a large part still remains insufficiently investigated, particularly its lentic habitats. In addition, the survey was conducted during the summer $(95 \%$ of records were collected from late June to mid-August). Future investigations will certainly add more species and give more precise distribution data for the region.

\section{ACKNOWLEDGEMENTS}

I would like to thank the reviewers for their constructive comments, which helped to improve the manuscript. This research was supported by Environmental Fund of the Federation of Bosnia and Herzegovina as part of the project „Identification of areas important for protection of threatened Odonata species in Federation of Bosnia and Herzegovina".

Received November 11, 2016

\section{REFERENCES}

Anonymous, 2012: Water Management Strategy of the Federation of Bosnia and Herzegovina, Zavod za vodoprivredu, Sarajevo and Mostar, pp 306.

Belančić, A., Bogdanović, T., Franković, M., Ljuština, M., Minoković, N. \& Vitas, B., 2008: Crvena knjiga vretenaca Hrvatske. Državni zavod za zaštitu prirode, Zagreb. [In Croatian with English summary].

Bognar, A., 2005: The Upper Part of the Una River Valley Between Martin Brod and Pritoka - Geomorphological Characteristics. Hrvatski geografski glasnik 67 (2), 21-38.

Boudot, J.P. \& Jović, M., 2015: Coenagrion scitulum (Rambur, 1842). In: Boudot, J.P., \& Kalkman, V.J. (eds.), Atlas of the European dragonflies and damselflies, KNNV Publishing, the Netherlands, p. 114-116.

Boudot, J.-P. \& Kuljer, D., 2015: Coenagrion ornatum (Selys, 1850). In: Boudot, J.-P. \& Kalkman, V.J. (eds.), Atlas of the European dragonflies and damselflies, KNNV Publishing, the Netherlands, p. 109-111.

Boudot, J.-P., Kalkman, V.J., Azpilicueta Amorín, M., Bogdanović, T., Cordero Rivera, A., Degabriele, G., Dommanget, J.-L., Ferreira, S., Garrigós, B., Jović, M., Kotarac, M., Lopau, W., Marinov, M., Minoković, N., Riservato, E., Samraoui, B. \& Schneider, W., 2009: Atlas of the Odonata of the Mediterranean and North Africa. Libellula. Supplement 9, 1-256.

De Knijf, G., TermaAt, T. \& Otт, J., 2015: Conservation of European dragonflies and damselflies. In: Boudot, J.-P. \& KalKman, V.J. (eds.), Atlas of the European dragonflies and damselflies, KNNV Publishing, the Netherlands, p. 27-35.

Dijkstra, K.-D.B. \& Lewington, R., 2006: Field guide to the Dragonflies of Britain and Europe. British Wildlife Publishing, Milton on Stour.

Franković, M. \& Vilenica, M., 2009: Studija inventarizacije vretenaca (Odonata) donjeg dijela rijeke Une i priobalnog pojasa. Državni zavod za zaštitu prirode, Zagreb, 1-14. [In Croatian].

Jović, M., Gligorović, B. \& Stanković, M., 2010: Review of faunistical data on Odonata in Bosnia and Herzegovina. Acta entomologica serbica 15 (1), 7-27.

International Sava River Basin Commission-ISRBC, 2009. Sava River Basin Analysis Report. International Sava River Basin Commission, Zagreb, pp 289.

Kalkman, V.J., Boudot, J.-P., Bernard, R., Conze, K.-J., De Knijf, G., Dyatlova, E., Ferreira, S., Jović, M., Ott, J., Riservato, E. \& Sahlen, G., 2010: European Red List of Dragonflies. Publications Office of the European Union, Luxembourg, pp 29. 
Kuljer, D., 2012: Odonata species and habitats at Livanjsko polje karst wetland area. IDF-Report. Newsletter of the International Dragonfly Fund 48, 1-38.

Kulijer, D., De Knijf, G. \& Franković, M., 2013: Review of the Odonata of Bosnia and Herzegovina. Odonatologica 42 (2), 109-123.

Kulijer, D., 2014: Odonata fauna of karst streams and rivers of South Herzegovina (Bosnia and Herzegovina, West Balkan). IDF-Report. Newsletter of the International Dragonfly Fund 72, 1-50.

Kuljer, D., 2015: Sympetrum flaveolum in the Dinaric Alps (Odonata: Libellulidae). Libellula 34 (1/2), 91-101.

Kulijer, D. \& Miljević, I., 2015: First record of Leucorrhinia caudalis for Bosnia and Herzegovina (Odonata: Libellulidae). Notulae odonatologicae 8 (6), 176-183.

Kulijer, D. \& Miljević, I., 2017: Dragonfly (Odonata) fauna of the Zelengora Mountain and National Park Sutjeska. Glasnik Šumarskog fakulteta Univerziteta u Banjoj Luci, 26. (in press)

Kulijer, D., Miljević, I. \& Jakovljev, J., 2016: Contribution of the participants of $4^{\text {th }}$ Balkan Odonatological Meeting to the knowledge of Odonata distribution in Bosnia and Herzegovina. IDF-Report. Newsletter of the International Dragonfly Fund 95, 1-25.

Kulijer, D., Vinko, D., Billqvist, M. \& Mekkes, J.J., 2012: Contribution to the knowledge of the Odonata fauna of Bosnia and Herzegovina - Results of the ECOO 2012. Natura Sloveniae 14 (2), 23-38.

Milanović, Đ., Stupar, V., Kulijer, D., Kotrošan, D. \& Hamzić A., 2015: Natura 2000 u Bosni i Hercegovini: dokle smo stigli?. Glasnik Šumarskog fakulteta Univerziteta u Banjoj Luci 23, 95-134. [In Serbian with English summary].

Riservato, E., Boudot, J.-P., Ferreira, S., Jović, M., Kalkman, V.J., Schneider, W., Samraoui, B. \& CutteLOD, A., 2009: The Status and Distribution of Dragonflies of the Mediterranean Basin, IUCN, Gland and Malaga, vii + pp 33 .

SluŽbeni GLASNik Republike SRPSKe, 2012: Uredba o Crvenoj listi zaštićenih vrsta flore i faune Republike Srpske. Službene novine Republike Srpske, 124/12, pp 54. [In Serbian].

Službeni list Federacije Bosne i Hercegovine, 2014: Crvena lista divljih vrsta i podvrsta biljaka, životinja i gljiva. Službeni list Bosne i Hercegovine, 7, p. 111-125. [In Bosnian].

ŠPanić, R., Cipčić, A., Bogdanović, T. \& Franković, M., 2011: State of research into the Dragonflies (Odonata) of Karlovac County, Croatia, with special reference to Natura 2000 species. Entomologia Croatica 15 (1-4), 209-221.

Vilenica, M. \& Dijkstra, K.-D.B., 2014: The Dragonfly (Insecta, Odonata) Fauna Of The Banovina Region, Croatia. Natura Croatica 23 (1), 45-66.

Vinko, D. \& Vilenica, M., 2013: BOOM 2013, 3rd Balkan Odonatological Meeting (Tretje mednarodno srečanje odonatologov Balkana) Hrvaška, 1.-7. avgust 2013: Rezultati. Erjavecia 28, 10-19.

\title{
SAŽETAK
}

\section{Prvi pregled faune vretenaca (Insecta, Odonata) sjeverozapadne Bosne}

\author{
D. Kulijer
}

Fauna vretenaca (Odonata) Bosne i Hercegovine je sve donedavno bila izuzetno slabo istražena. Tek su zadnjih desetak godina započeta prva detaljnija istraživanja ovih kukaca, što je rezultiralo velikim brojem novih podataka i većim brojem publikacija. I pored značajnog napretka u poznavanju rasprostranjenosti vrsta, neka područja zemlje su i dalje ostala slabo istražena. To se posebno odnosi na sjeverozapadni dio Bosne, za koji gotovo da nisu postojali podaci o fauni ovih kukaca.

Cilj rada bio je istražiti faunu vretenaca sjeverozapadne Bosne u cilju stjecanja što bolje slike o njihovoj fauni na području sliva rijeke Une, kojeg dijele Bosna i Hercegovina i Hrvatska.

Podaci su prikupljeni terenskim istraživanjima tijekom 2012. (26.-27.06. i 19.-20.08.) i 2013. (25.-29.07.) godine. Osim toga, analizirani su i podaci iz dostupnih publikacija, entomoloških 
kolekcija Zemaljskog muzeja Bosne i Hercegovine i baze podataka vretenaca BiH. Istraživanje je uglavnom obuhvatilo odrasle jedinke, dok su ličinke i svlakovi sakupljani sporadično. Područje istraživanja je sjeverozapadni dio Bosne i Hercegovine, između $15^{\circ} 43^{\prime}$ i $16^{\circ} 32^{\prime}$ istočne geografske dužine i $44^{\circ} 09^{\prime}$ i $45^{\circ} 13^{\prime}$ sjeverne geografske širine. Ovo područje obuhvaća najveći dio sliva rijeke Une, kao i male dijelove slivova rijeka Korane i Gline koji se nalaze na teritoriju $\mathrm{BiH}$.

Prikupljeno je ukupno 275 nalaza 36 vrsta (56 \% faune Odonata $\mathrm{BiH}$ ) sa 75 različitih lokacija. Najznačajniji su nalazi dvije vrste sa Dodatka II Direktive o staništima Europske unije: Coenagrion ornatum (Selys, 1850) i Cordulegaster heros Theischinger, 1979, kao i pronalasci vrsta Chalcolestes viridis (Vander Linden, 1825) i Coenagrion scitulum (Rambur, 1842) koje se na temelju postojećih podataka smatraju rijetkima u Bosni i Hercegovini. Tijekom ovog istraživanja C. viridis je zabilježen na lokalitetu Vedro polje kod Bihaća, dok je vrsta C. scitulum pronađena u južnom dijelu područja istraživanja na Pašića, Petrovačkom i Bjelajskom polju.

Vrsta C. heros je zabilježena na osam lokaliteta te se čini da je na ovom području rasprostranjena, posebno u njegovom sjeveroistočnom dijelu. Coenagrion ornatum je zabilježen jedino na Bjelajskom i Petrovačkom polju. No, s obzirom da se radi o proljetnoj vrsti, te da ovo područje u tom periodu nije bilo istraživano, moguće je da je ona i znatno šire rasprostranjena, posebno u krškim poljima i nižim dijelovima na sjeveru i istoku regije. Najveći broj nalaza ove vrste $\mathrm{u} \mathrm{BiH}$ vezan je upravo za krška polja. $\mathrm{S}$ obzirom da su vrste $C$. ornatum i $C$. heros navedene u Dodatku II Direktive o staništima Europske unije, te da je C. heros također ranjiva (VU) vrsta na Crvenoj listi Odonata Mediterana, u budućnosti im treba posvetiti posebnu pažnju, prije svega u procesu identifikacije područja za mrežu Natura 2000 u BiH.

Po brojnosti lokaliteta primjetna je dominacija reofilnih vrsta, što je i očekivano s obzirom na značajnu zastupljenost potoka i manjih rijeka među istraživanim lokalitetima. Proljetne vrste i vrste koje primarno naseljavaju veće stajaćice su bile rijetke ili uopće nisu zabilježene. Među vrstama koje nisu zabilježene ovim istraživanjem nalaze se i neke koje su relativno česte u okolnim regijama BiH i Hrvatske. Razlog za njihovu neprisutnost je s jedne strane bolja istraženost okolnih područja, a s druge strane nedovoljna pokrivenost različitih sezonskih aspekata i mali broj većih stajaćih voda tijekom istraživanja.

Ovo istraživanje predstavlja značajan doprinos poznavanju vretenaca sliva rijeke Une i Bosne i Hercegovine. S obzirom na veličinu i kompleksnost istraživanog prostora, kao i na djelomičnu pokrivenost po pitanju različitih sezonskih aspekata i staništa, buduća istraživanja će sigurno rezultirati novim vrstama za ovo područje. 